\title{
Gamble on gaze: Eye movements reflect the numerical value of blackjack hands
}

\author{
Kevin J. Holmes ${ }^{1}$ - Vladislav Ayzenberg ${ }^{2}$ - Stella F. Lourenco ${ }^{2}$
}

Published online: 12 May 2016

(C) Psychonomic Society, Inc. 2016

\begin{abstract}
We used a novel task - a blackjack game that naturally involves mental summation of numerical values - to investigate the role of attention in the mental number line (MNL) and to provide insight into the ecological validity of this representational format. By analyzing the spatial position of participants' spontaneous, task-irrelevant eye movements, we avoided some of the limitations of previous research on the $\mathrm{MNL}$, in which the findings could be attributed to taskspecific factors such as the use of overt spatial cues. In two experiments, we found that eye movements along the horizontal axis reflected the overall numerical value of participants' hands, with smaller-value hands eliciting fixations toward the left of the screen and larger-value hands eliciting fixations toward the right. This pattern held even when controlling for the number of cards in the hand and the value of the card most recently dealt-suggesting that the effects were driven by mental summation of values, not merely by the processing of serial order or individual numbers. Vertical eye movements, in contrast, reflected hand value less reliably. In showing that spontaneous eye movements along the horizontal axis track the magnitude of internally computed sums in an ecologically relevant task, our findings provide evidence for a dynamic
\end{abstract}

Electronic supplementary material The online version of this article (doi:10.3758/s13423-016-1055-0) contains supplementary material, which is available to authorized users.

Kevin J. Holmes

kjholmes@coloradocollege.edu

Stella F. Lourenco

stella.lourenco@emory.edu

1 Department of Psychology, Colorado College, 14 E. Cache La Poudre St., Colorado Springs, CO 80903, USA

2 Department of Psychology, Emory University, 36 Eagle Row, Atlanta, GA 30322, USA
MNL that supports magnitude-driven shifts of attention and that may be recruited during everyday forms of numerical reasoning.

Keywords Mental number line $\cdot$ Eye movements $\cdot$ Mental arithmetic $\cdot$ Spatial representation $\cdot$ Blackjack

In blackjack, players must repeatedly decide whether to stick with the cards in their hand or risk a "bust" by taking another card (Keren \& Wagenaar, 1985). Mentally summing the values of successive cards, and thereby computing the total value of one's hand, is a prerequisite for making this decision wisely. Research suggests that such mental arithmetic may rely on an internal spatial representation of numerical magnitude (e.g., Knops, Viarouge, \& Dehaene, 2009; Marghetis, Nuñez, \& Bergen, 2014; McCrink, Dehaene, \& DehaeneLambertz, 2007; for a review, see Fischer \& Shaki, 2014). For example, when pointing to an arithmetic solution on a visually presented number line, participants are biased leftward on subtraction problems and rightward on addition problems (Pinhas \& Fischer, 2008). Such spatial biases suggest that mental arithmetic may induce dynamic shifts of attention, or simulated movement, along a left-to-right-oriented mental number line (Knops, Dehaene, Berteletti, \& Zorzi, 2014; Marghetis et al., 2014; Masson \& Pesenti, 2014; McCrink et al., 2007), building on earlier findings from number classification tasks in which smaller and larger numbers yielded faster left- and right-side responses, respectively (e.g., Dehaene, Bossini, \& Giraux, 1993).

Although the notion of a dynamic mental number line (MNL) is intuitively appealing, there are alternative explanations for the spatial biases observed in both number classification and mental arithmetic tasks. In particular, such biases may be due to task-specific demands imposed by the overtly 
spatial nature of the experimental procedure (Santiago \& Lakens, 2015). In many studies, participants respond along a particular spatial axis-for example, by pressing left- and right-side response keys (e.g., Dehaene et al., 1993; Pinhas \& Fischer, 2008) or by making leftward and rightward head or arm movements (e.g., Loetscher, Schwarz, Schubiger, \& Brugger, 2008; Marghetis et al., 2014; Wiemers, Bekkering, \& Lindemann, 2014; Winter \& Matlock, 2013). In other studies, the numerical stimuli themselves are presented at different spatial locations (e.g., Knops, Viarouge et al., 2009; Masson $\&$ Pesenti, 2014). Such methods leave open the possibility that the observed biases are artifacts of polarity correspondence (e.g., left-less/right-more, a type of stimulus-response compatibility; Proctor \& Cho, 2006), rather than evidence of an MNL (see also Santens \& Gevers, 2008). Moreover, in the case of mental arithmetic specifically, spatial biases may be driven by semantic associations between space and arithmetic operations (e.g., left-subtraction/right-addition; Hartmann, Mast, \& Fischer, 2015), not by shifts of attention along the MNL toward the outcome of such operations (i.e., solution magnitude; cf. Marghetis et al., 2014). Thus, previous research, though suggestive, has not provided unambiguous evidence for spatial representations of number along which movement is mentally simulated.

In addition to these interpretive concerns, the tasks used in MNL studies may be criticized on the grounds of ecological validity. The standard paradigms, in which participants make binary judgments about the parity or magnitude of isolated digits (e.g., Dehaene et al., 1993), are notably artificial. Even the stimuli used in research on mental arithmetic (e.g., Pinhas $\&$ Fischer, 2008), though reflective of arithmetic facts in formal schooling, are far from generalizable to the plethora of numerical problems encountered in everyday settings. Showing that spatial biases play a role in real-world numerical decisions, like whether to "hit" or "stay" in blackjack, would help to establish the ecological validity of these effects.

In the present experiments, we addressed issues of interpretation and generalizability by investigating spatial biases in a blackjack task. Participants played a version of blackjack in which they were shown successive, centrally presented playing cards and decided to hit or stay-a decision that naturally involves mental arithmetic (here, addition; for standard blackjack rules, see Keren \& Wagenaar, 1985). To avoid priming spatial biases via explicit cues (e.g., left- and right-side responses), we used a go/no-go task in which participants pressed a central response key (to hit) or withheld responding (to stay). We analyzed participants' spontaneous eye movements on a blank screen following the presentation of each card. Eye movements have been used to study diverse cognitive processes, both in the lab and in more natural settings (e.g., Hayhoe \& Ballard, 2005; Loftus \& Mackworth, 1978), and, importantly, have provided insight into mental associations between space and number (e.g., Bulf, de Hevia, \&
Cassia, 2015; Hartmann et al., 2015; Klein, Huber, Nuerk, \& Moeller, 2014; Knops, Thirion, Hubbard, Michel, \& Dehaene, 2009).

For our purposes, eye movements were assessed in order to determine whether they reflected the total value of participants' blackjack hands (i.e., the outcome of mental addition). On the basis of evidence for left-to-right orientation of number in Westerners, and that gaze patterns reflect this orientation (Hartmann et al., 2015; Loetscher, Bockisch, \& Brugger, 2008; Loetscher, Bockisch, Nicholls, \& Brugger, 2010), we expected that smaller-value and larger-value hands would elicit more leftward and rightward eye movements, respectively. Because vertical orientation of number has also been observed (e.g., Hartmann, Gashaj, Stahnke, \& Mast, 2014; Loetscher et al., 2010; Winter \& Matlock, 2013; for discussion of mixed findings, see Hartmann et al., 2014; Holmes \& Lourenco, 2012), we assessed whether upward and downward eye movements also reflected the value of participants' hands.

This task design avoided some of the limitations of previous research. Since both the stimuli and responses were centrally located and eye movements were task-irrelevant, the task had no overt spatial component, thus precluding any interpretation based on polarity correspondence. Moreover, spatial biases in mental arithmetic are typically based on a comparison of addition and subtraction operations (e.g., Marghetis et al., 2014; Pinhas \& Fischer, 2008; Wiemers et al., 2014). In our task, operation was held constant (blackjack requires addition, but not subtraction, of values); thus, any relation between gaze patterns and hand value would reflect spatial-numerical mappings, not semantic associations based on operation alone (cf. Hartmann et al., 2015). Finally, our experiments assessed the kind of numerical thinking that might be involved when playing blackjack in a real-world setting, and hence might be more generalizable than other MNL studies.

\section{Experiment 1}

In our first experiment, we assessed eye fixations following the presentation of each playing card, before participants decided to hit or stay. Of particular interest was whether the fixations would reflect the current total value of the hand (i.e., the solution magnitude), as opposed to the value of any single card (i.e., the operands). Consider, for example, a hand in which the player is dealt a 2 , followed by a 10 . The total hand value (12) is toward the low end of the range of viable two-card hands (i.e., 4 to 21), but the value of the most recently dealt card (10) is on the high end of the range of single cards (i.e., 2 to 11). If horizontal fixations reflect the total hand value rather than the value of the last card presented, they should be more leftward (i.e., relatively low value) for this hand. Such a pattern would suggest that eye movements in this task reflect 
mental arithmetic, not just the processing of individual numbers.

\section{Method}

Participants Thirty undergraduates (21 female, nine male) participated for course credit, a sample size chosen to enable detection of a medium-to-large effect size, as has been found in comparable studies (e.g., Hartmann et al., 2015; Loetscher et al., 2010). All participants had normal or corrected-tonormal vision. The majority (27) were right-handed $(M=$ 62.3, ranging from -100 to 100 according to the Edinburgh Handedness Inventory; Oldfield, 1971). Procedures were approved by the Institutional Review Board (IRB).

Materials and equipment The stimuli were 52 rectangular images of playing cards $\left(6.9^{\circ} \times 1.1^{\circ}\right)$, as in a standard deck (i.e., cards 2 through ace for each of the four suits), and were displayed on a 17-in. eyetracker monitor (Tobii ET-17; 1,024 $\times 768$ resolution, sampling rate $=30 \mathrm{~Hz}$ ) at a viewing distance of $60 \mathrm{~cm}$.

Procedure Participants sat in a darkened room and were encouraged to remain still and maintain attention on the screen. After a 5-point eyetracker calibration, participants played a blackjack game presented onscreen. The task consisted of a series of solo blackjack hands, with participants accumulating points equaling the total value of the cards in their hand (up to 21). No points were earned for hands exceeding 21 . Participants were instructed to treat face cards (i.e., the jack, queen, and king) as representing a value of 10 , and aces a value of 11. They were encouraged to accumulate as many points as possible during the experiment, but were not otherwise incentivized.

At the start of each trial, participants clicked on the "DEAL" button to deal the first card, and the trial proceeded as is shown in Fig. 1. Three pairs of countdown lights signaled participants to either "hit" (i.e., deal another card) or "stay" (i.e., end the trial and bank the accumulated points). When the screen turned blue, participants had $3 \mathrm{~s}$ to make a go/no-go response, pressing the space bar to hit or nothing to stay. Following a hit, another card was dealt immediately. If this card yielded a total hand value less than 21, the same sequence of events was repeated for the new card. If the new card yielded a total hand value exceeding 21 , feedback was provided, and the next trial began $3 \mathrm{~s}$ later. Following a stay, the next trial also began $3 \mathrm{~s}$ later.

There were six practice trials and 52 test trials, presented in one of four randomized orders. The order of the cards within a given trial was pseudorandom, to ensure sufficient trials with three- or four-card hands and no trials with hands of more than four cards.

\section{Results and discussion}

On average, participants scored 19.3 points $(S D=0.9)$ per trial (i.e., close to the target of 21 without going over), and all scored between 16 and 21 per trial, consistent with normative blackjack strategy (Keren \& Wagenaar, 1985) and confirming engagement with the task. ${ }^{1}$ Analyses focused on fixations on the monitor during the 3-s window following the initial presentation of each card, when participants were presumably computing the value of their hands (i.e., mentally adding the current card's value to that of any previous cards) and no stimuli were onscreen (see Fig. 1). ${ }^{2}$ For a given card, the mean horizontal $(x)$ and vertical $(y)$ coordinates (in degrees of visual angle from the screen center) were computed by averaging across all recorded fixations during this window, excluding those greater than $2.5 \mathrm{SDs}$ from individual participant means ( $2.1 \%$ and $2.6 \%$ of fixations, respectively).

To determine whether the horizontal and vertical fixations reflected hand value, the $x$ - and $y$-coordinate means were regressed on the total hand values ( 2 through 21$)^{3}$ for each participant (see Table S1 in the supplemental material for the numbers of trials by value). This yielded unstandardized slope coefficients, with positive slopes indicating left-to-right orientation and bottom-to-top orientation of increasing values, respectively (and negative slopes indicating the reverse orientations). The $x$-coordinate slope was positive and differed significantly from zero $(M=0.021 \%$ value, $S D=0.026,95 \% \mathrm{CI}$ : $0.011,0.031), t(29)=4.28, p=.0002, d=0.78$, indicating leftto-right orientation, whereas the $y$-coordinate slope was negative and also differed significantly from zero $(M=-0.034 \%$ value, $S D=0.046,95 \% \mathrm{CI}:-0.051,-0.017), t(29)=4.02, p=$ $.0004, d=0.73$, indicating top-to-bottom orientation. Moreover, 21 of 30 participants had a positive $x$-coordinate slope, and 27 of 30 had a negative $y$-coordinate slope; both proportions were significantly greater than chance (sign tests: $p$ s <.05), suggesting similar results across participants (see Fig. S1 in the supplemental material for the individual participant slopes). These results indicate that smaller-value hands elicited fixations toward the left and top of the screen, and larger-value hands elicited fixations toward the right and bottom of the screen (i.e., left-to-right and top-to-bottom orientation).

\footnotetext{
${ }^{1}$ The mean number of points scored per trial was unrelated to any of the gaze measures reported in either experiment.

${ }^{2}$ Across both experiments, $17.4 \%$ of the samples contained no recorded fixations. In analyses of eye movements during the early and late halves of the 3-s interval (cf. van Dijck \& Fias, 2011), both halves showed the same pattern that we found overall.

${ }^{3}$ The total hand value generally corresponded to the quality of the hand, but not always (e.g., a hand of 11 is stronger than a hand of 12 because the chance of a "bust" is smaller). Fixations did not appear to reflect quality more than value; low-quality hands (e.g., 12-15) did not deviate in any systematic way from the overall trends (see Figs. 2, 3, and 5).
} 


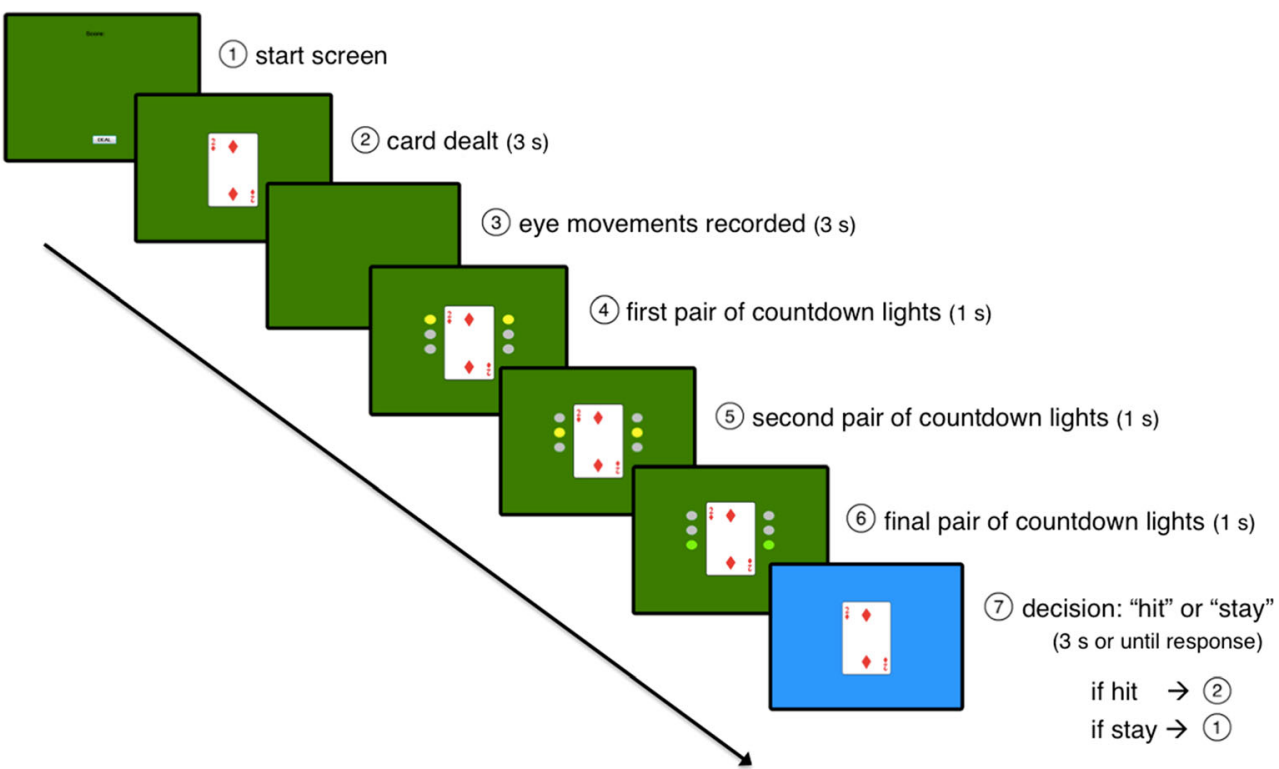

Fig. 1 Sequence of events on each trial in Experiment 1

Importantly, these gaze patterns were not driven by serialorder processing of the number of cards in the hand (cf. Rinaldi, Brugger, Bockisch, Bertolini, \& Girelli, 2015). After partialing out the number of cards, the total hand value remained a significant predictor of both $x$-coordinates $(M=$ $0.022 \%$ value, $S D=0.030,95 \%$ CI: $0.011,0.033), t(29)=$ $4.11, p=.0003, d=0.75$, and $y$-coordinates $(M=-0.032 \%$ value, $S D=0.042,95 \% \mathrm{CI}:-0.048,-0.017), t(29)=4.19, p=$ $.0002, d=0.76$. Additional analyses using Bonferroni correction (alpha $=.025$ ) showed that left-to-right orientation held throughout a given hand, and thus did not depend on the elapsed time within a trial. The $x$-coordinate slopes were significantly positive both early in the hand (i.e., following the first and second cards dealt; $M=0.023 \%$ value, $S D=0.027$, $95 \%$ CI: $0.013,0.033), t(29)=4.59, p<.0001, d=0.84$, and late in the hand (i.e., following the third and fourth cards dealt; $M=0.022^{\circ}$ /value, $S D=0.043,95 \%$ CI: $\left.0.006,0.038\right), t(29)=$ $2.84, p=.008, d=0.52$ (see Fig. 2). In contrast, top-to-bottom orientation was less consistent. Although the $y$-coordinate slopes were significantly negative early in the hand $(M=-$ $0.035 \%$ value, $S D=0.050,95 \%$ CI: $-0.054,-0.016), t(29)=$ $3.83, p=.0006, d=0.70$, they did not differ significantly from zero late in the hand $(M=-0.006 \%$ value, $S D=0.065,95 \%$ CI: $-0.030,0.019), t(29)=0.47, p>.6$ (see Fig. 3$){ }^{4}$

Although the analyses above showed that gaze patterns reflect total hand value, these effects may have been driven largely by the value of the last card presented (i.e., the number shown just prior to the eye movements). To address this possibility, we computed a new set of slopes for each participant,

\footnotetext{
${ }^{4}$ Slopes were comparable across the two halves of the experiment. Together with the lack of overt spatial cues, this suggests the activation of an MNL stored in long-term memory, as opposed to an ad-hoc spatial representation driven by task demands (cf. Fias, 2015).
}

regressing their $x$ - and $y$-coordinates on the total hand value after partialing out the last card's value. The $x$-coordinate slopes remained significantly positive $(M=0.021 \%$ value, $S D$ $=0.031,95 \%$ CI: 0.009, 0.033), $t(29)=3.68, p=.0009, d=$ 0.67 , and the $y$-coordinate slopes remained significantly negative $(M=-0.033 \%$ value, $S D=0.056,95 \% \mathrm{CI}:-0.054$, $0.012), t(29)=3.26, p=.003, d=0.59$. Thus, both the horizontal and vertical gaze patterns reflected the total hand value, not just the value of the last card presented.

These results suggest that the numerical value of blackjack hands is revealed by spontaneous eye movements, with total hand value increasing from left to right and from top to bottom. The latter effect contrasts with those observed in previous studies, in which values were generally found to increase from bottom to top rather than the reverse (e.g., Holmes \& Lourenco, 2012; Loetscher et al., 2010; but see Hung et al.,

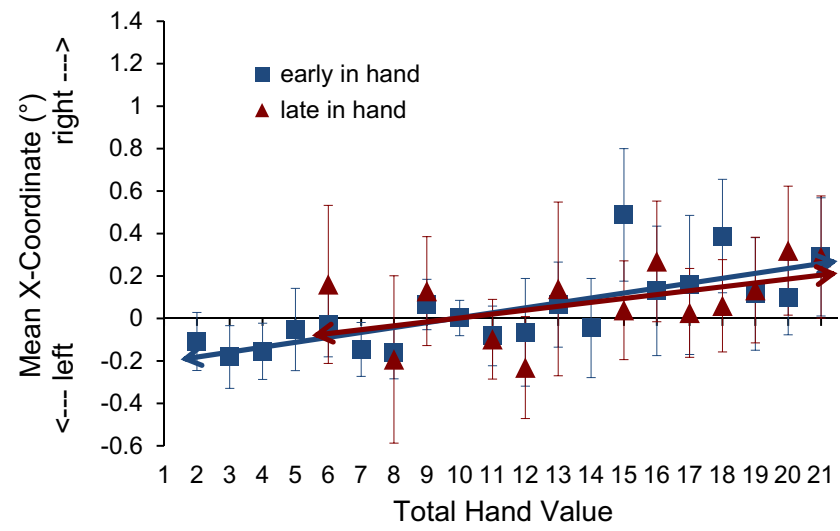

Fig. 2 Mean $x$-coordinates (in degrees) by total hand value in Experiment 1 for fixations early in the hand (first and second cards dealt; squares) and late in the hand (third and fourth cards dealt; triangles), with $0^{\circ}$ indicating the horizontal midpoint on the screen. Error bars show $95 \%$ within-participants confidence intervals 


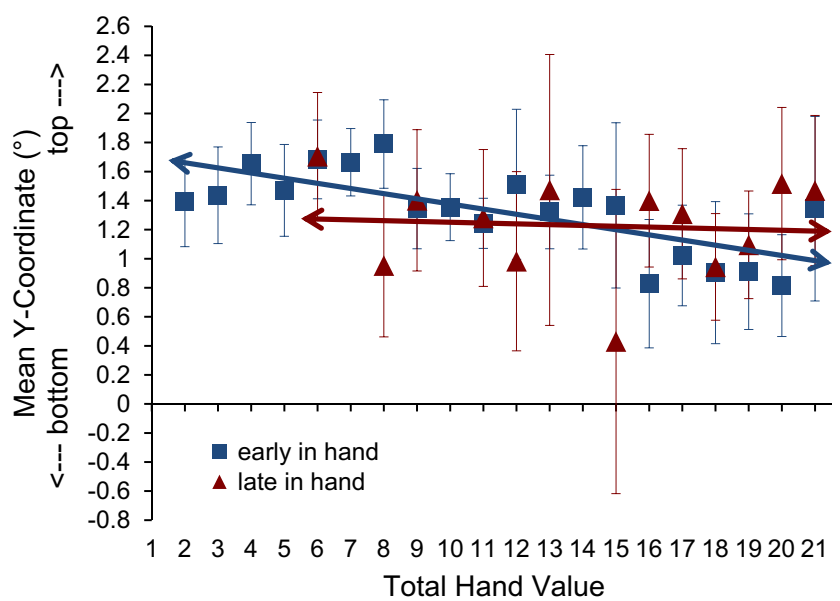

Fig. 3 Mean $y$-coordinates (in degrees) by total hand value in Experiment 1 for fixations early in the hand (first and second cards dealt; squares) and late in the hand (third and fourth cards dealt; triangles), with $0^{\circ}$ indicating the vertical midpoint on the screen. Error bars show $95 \%$ within-participants confidence intervals. Fixations were generally located above the vertical midpoint, perhaps reflecting a tendency to look upward during problem solving, at least in Westerners (McCarthy, Lee, Itakura, \& Muir, 2006)

2008). However, the slope analyses showed that only the horizontal effect was stable across both early and late points in the hand, suggesting that the vertical effect might be less robust. Moreover, two aspects of the procedure may have primed a top-to-bottom orientation inadvertently. First, the countdown lights prior to the response were illuminated from top to bottom (see Fig. 1); this may have encouraged participants to adopt a top-to-bottom scanning pattern that primed the corresponding orientation of numerical values (cf. Shaki \& Fischer, 2008). Second, the numbers on the card stimuli were at the top left and bottom right, also possibly prompting top-to-bottom (and left-to-right) scanning patterns and the corresponding spatial-numerical associations. These confounds were eliminated in Experiment 2 to provide a purer test of gaze patterns during blackjack.

\section{Experiment 2}

Here we used fully symmetrical countdown lights and card stimuli to avoid any task-induced scanning patterns. We expected that vertical eye movements would now reflect hand value only weakly, if at all (cf. Gevers, Lammertyn, Notebaert, Verguts, \& Fias, 2006; Holmes \& Lourenco, 2012).

\section{Method}

Participants Twenty-eight undergraduates (21 female, seven male) participated for course credit. All had normal or corrected-to-normal vision, and the majority (25) were right- handed ( $M=62.1$, ranging from -100 to 100$)$. All procedures were IRB-approved.

Materials and procedure The card stimuli were modified to be fully symmetrical (see Fig. 4). The procedure was identical to that of Experiment 1, with one exception: The countdown lights were displayed symmetrically, all showing the same color (red, yellow, or green; see Fig. 4).

\section{Results and discussion}

On average, participants scored 18.1 points $(S D=2.4)$ per trial, and the majority ( 24 of 28 ) scored between 16 and 21 per trial, confirming task engagement. As in Experiment 1, the mean $x$ - and $y$-coordinates of fixations during the 3 -s window following each card were computed $(1.7 \%$ and $3.0 \%$ of fixations were excluded, respectively) and regressed on the total hand values to produce slopes (see Table S1 in the supplemental material for the number of trials by value). The $x$-coordinate slopes were significantly positive $\left(M=0.028^{\circ} /\right.$ value, $S D=0.028,95 \%$ CI: $0.017,0.038), t(27)=5.24, p<$ $.0001, d=0.99$, indicating left-to-right orientation, and the $y$-coordinate slopes were significantly negative $(M=$ $-0.019^{\circ}$ value, $S D=0.036,95 \%$ CI: $\left.-0.032,-0.005\right)$, $t(27)=2.75, p=.01, d=0.52$, indicating top-tobottom orientation. Moreover, 23 of 28 participants had positive $x$-coordinate slopes, and 22 of 28 had negative $y$-coordinate slopes, with both proportions being significantly greater than chance (sign tests: $p \mathrm{~s}<.05$; see Fig. S2 in the supplemental material for the individual participant slopes).

After partialing out the number of cards in the hand, the total hand value remained a significant predictor of both $x$ coordinates $\left(M=0.019^{\circ} /\right.$ value, $S D=0.032,95 \%$ CI: 0.007 , $0.031), t(27)=3.15, p=.004, d=0.60$, and $y$-coordinates $(M$ $=-0.026 \%$ value, $S D=0.059,95 \%$ CI: $-0.049,-0.003), t(27)$ $=2.32, p=.03, d=0.44$, suggesting that the gaze patterns were not driven by serial-order processing of the card number. When broken down by early versus late stages of the hand (Bonferroni corrected), left-to-right orientation was significant early in the hand $(M=0.017 \%$ value, $S D=0.025,95 \% \mathrm{CI}$ : $0.007,0.026), t(27)=3.54, p=.002, d=0.67$, and approached significance late in the hand $\left(M=0.033^{\circ}\right.$ value, $S D=0.088$, $95 \%$ CI: $-0.001,0.067), t(27)=1.98, p=.058, d=0.37$; see Fig. 5). In contrast, top-to-bottom orientation did not reach significance either early or late $(p s>.025 ; 95 \%$ CIs: early $=-0.034,-0.002$; late $=-0.098,-0.005$ ). These results suggest that hand value is reflected in horizontal eye movements, but less so in vertical ones.

This conclusion is further supported by analyses of the fixations after partialing out the value of the last card presented. The $x$-coordinate slopes remained significantly positive ( $M$ 


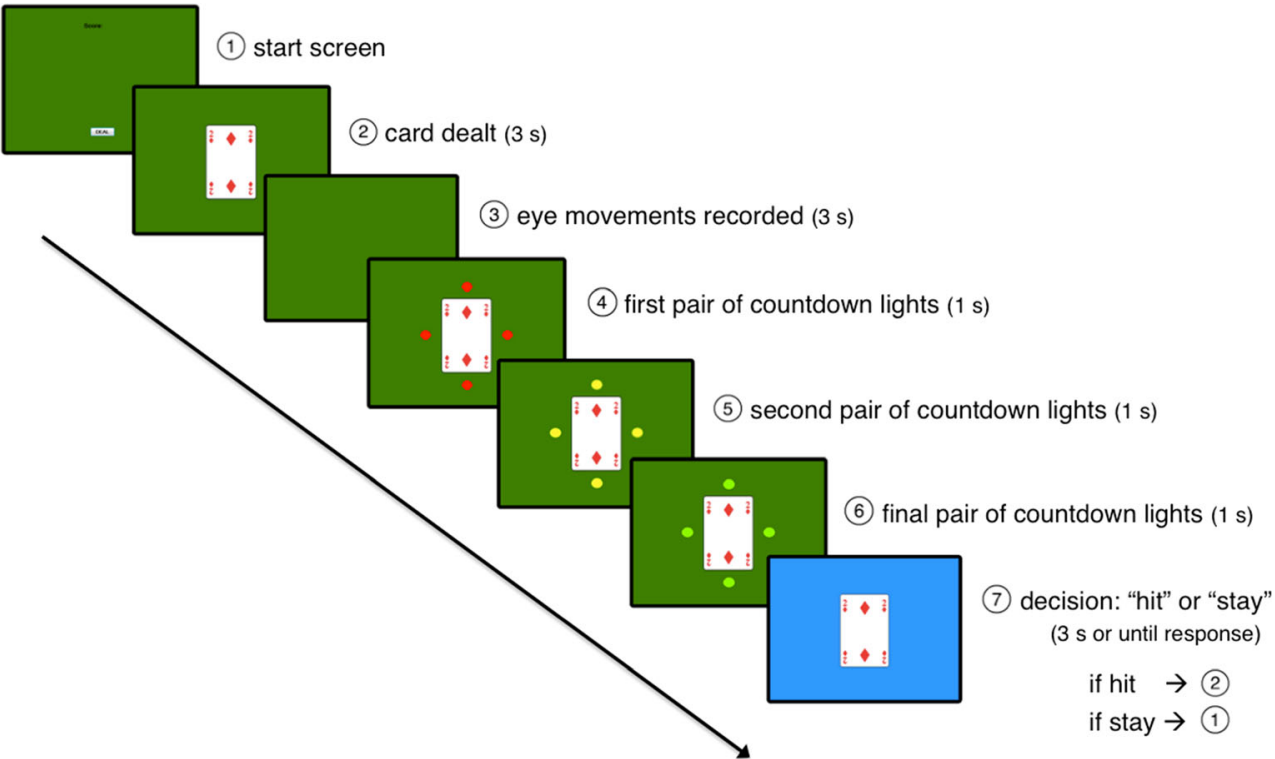

Fig. 4 Sequence of events on each trial in Experiment 2

$=0.038^{\circ}$ /value, $S D=0.035,95 \%$ CI: $\left.0.025,0.052\right), t(27)=$ $5.85, p<.0001, d=1.11$, replicating Experiment 1, but the $y$ coordinate slopes did not $\left(M=-0.010^{\circ}\right.$ /value, $S D=0.039$, $95 \%$ CI: $-0.025,0.005), t(27)=1.31, p>.2$. Thus, after eliminating procedural confounds that may have primed vertical eye movements, horizontal gaze patterns continued to reflect the total hand value and were not driven merely by the last card's value, whereas vertical gaze patterns were weaker and driven solely by the last card's value. Moreover, the observed top-to-bottom orientation is surprising, given the opposite orientation (bottom-to-top) found in previous studies (e.g., Holmes \& Lourenco, 2012; Loetscher et al., 2010). Hung et al. (2008) found top-to-bottom orientation in Chinese speakers when processing Chinese number words, but our English-speaking participants had no particular experience, linguistic or otherwise, that might be expected to yield

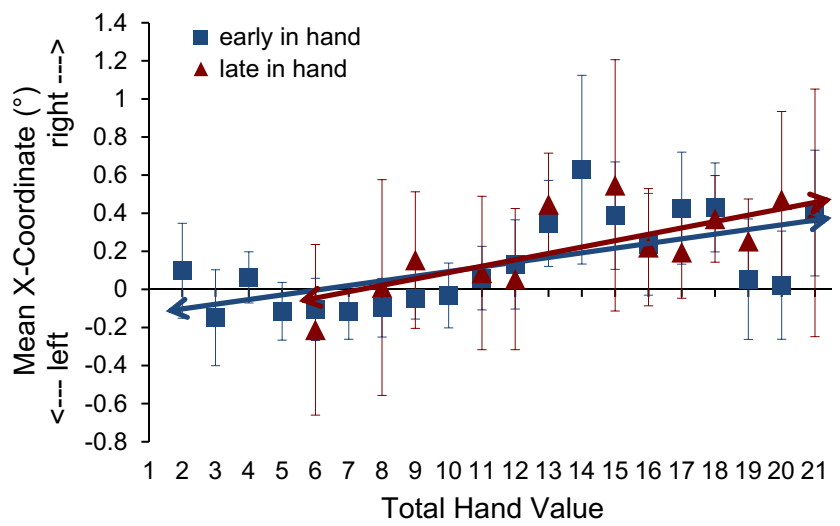

Fig. 5 Mean $x$-coordinates (in degrees) by total hand value in Experiment 2 for fixations early in the hand (first and second cards dealt; squares) and late in the hand (third and fourth cards dealt; triangles), with $0^{\circ}$ indicating the horizontal midpoint on the screen. Error bars show $95 \%$ within-participants confidence intervals such an orientation. Future research will be needed to assess the reliability of the vertical effect and its potential sources.

\section{General discussion}

Our findings show that spontaneous eye movements during a blackjack game reflect the numerical value of players' hands. Smaller-value hands elicited leftward fixations, and largervalue hands elicited rightward fixations. These effects were driven by the total hand value: Even when controlling for the number of cards in the hand and the value of the card just dealt, the overall value of the hand predicted the horizontal position of participants' gaze. This suggests that the horizontal eye movements in our task reflect the mental summation of values, not merely the processing of serial order or individual numbers. The results were less clear for vertical eye movements. Although smaller- and larger-value hands tended to produce upward and downward fixations, respectively, these effects were less reliable than their horizontal counterparts, and upon removal of procedural confounds, were driven solely by the last card's value - not by summation of multiple values. In summary, our results show that mental arithmetic during blackjack elicits horizontal shifts of attention that track the magnitude of those internally computed sums. These findings are consistent with a spatial representation of number that supports dynamic attentional shifts toward computed magnitudes.

Previous studies of spatial biases in mental arithmetic have relied on a comparison of addition and subtraction, with leftward biases observed for subtraction and rightward biases for addition, irrespective of the solution - an effect dubbed operational momentum (OM; Hartmann et al., 2015; 
Marghetis et al., 2014; Masson \& Pesenti, 2014; Pinhas \& Fischer, 2008). Our findings show that spatial biases in mental arithmetic also reflect the solution magnitude (i.e., total hand value), suggesting sensitivity to the outcome - not just the direction - of the operation, at least when operation is held constant. Although several studies have reported similar effects of solution magnitude (e.g., more rightward responses for problems with larger solutions; Marghetis et al., 2014; Masson \& Pesenti, 2014; Pinhas \& Fischer, 2008) in addition to OM effects, these studies used procedures with overt spatial cues, which, as we discussed above, may not generalize to nonspatial contexts. In showing that eye movements reflect total hand value in the absence of explicit cues, our findings provide support for such generalizability.

Perhaps most directly comparable to our findings are those of Hartmann et al. (2015), who also examined spatial biases in mental arithmetic using eye movements on a blank screen, but for both addition and subtraction. Hartmann et al. observed spatial biases only for vertical eye movements, and showed that this effect was driven specifically by the operator (i.e., plus/minus), not by the value of the operands or solution magnitude - and hence not by the computational process of mental arithmetic. These results were interpreted as evidence against the notion of dynamic attentional shifts along the MNL. In showing effects of solution magnitude independent of operator, our findings suggest that mental arithmetic (specifically, continuous summation) does yield attentional shifts - and along the horizontal axis rather than the vertical.

What accounts for the discrepancies between these findings? One possibility is that the differences are due to the greater complexity of our blackjack task. Unlike the relatively simple two-operand problems used by Hartmann et al. (2015), for which the retrieval of stored solutions or the activation of streamlined procedures might have sufficed (Fayol \& Thevenot, 2012), our participants often mentally added more than two operands, presented in the relatively atypical format of playing cards. These built-in features of blackjack may yield spatial biases attributable specifically to computation. Moreover, the vertical effects observed by Hartmann et al. may have depended on the semantic contrast between addition and subtraction (i.e., dichotomous "more is up"/"less is down" mappings); in the absence of this contrast and given a task that is sufficiently complex, horizontal biases driven by solution magnitude may emerge.

Our findings highlight the value of using ecologically relevant stimuli to investigate spatial representations of number. Such stimuli may inform the extent to which the cognitive processes identified by more traditional methods are likely to manifest in real-world settings. Of course, we acknowledge that our blackjack task is in many respects unlike a real blackjack game, and that the relatively small differences in absolute gaze position shown here may be undetectable to the naïve observer. Future research should explore whether observers can, perhaps following training, rely on gaze patterns to infer hand value, as well as how spatial representations - already shown to extend beyond number to other magnitudes (e.g., Fischer, Riello, Giordano, \& Rusconi, 2013; Fumarola et al., 2014; Holmes \& Lourenco, 2011, 2013)—may support everyday quantitative reasoning more generally.

Author note Preparation of the manuscript was supported in part by a Faculty Opportunity Grant from Colorado College. We thank Eileen Kitrick for assistance with figure design and three anonymous reviewers for helpful feedback.

\section{References}

Bulf, H., de Hevia, M. D., \& Cassia, V. M. (2015). Small on the left, large on the right: Numbers orient visual attention onto space in preverbal infants. Developmental Science, 19, 394-401. doi:10.1111/desc. 12315

Dehaene, S., Bossini, S., \& Giraux, P. (1993). The mental representation of parity and number magnitude. Journal of Experimental Psychology: General, 122, 371-396. doi:10.1037/0096-3445.122. 3.371

Fayol, M., \& Thevenot, C. (2012). The use of procedural knowledge in simple addition and subtraction problems. Cognition, 123, 392-403. doi:10.1016/j.cognition.2012.02.008

Fias, W. (2015). Neurocognitive components of mathematical skills and dyscalculia. In D. B. Berch, D. C. Geary, \& K. M. Koepke (Eds.), Development of mathematical cognition: Neural substrates and genetic influences (Vol. 2, pp. 195-217). San Diego, CA: Elsevier.

Fischer, M. H., Riello, M., Giordano, B. L., \& Rusconi, E. (2013). Singing numbers ... in cognitive space - A dual-task study of the link between pitch, space, and numbers. Topics in Cognitive Science, 5, 354-366. doi:10.1111/tops. 12017

Fischer, M. H., \& Shaki, S. (2014). Spatial associations in numerical cognition-From single digits to arithmetic. Quarterly Journal of Experimental Psychology, 67, 1461-1483. doi:10.1080/17470218. 2014.927515

Fumarola, A., Prpic, V., Da Pos, O., Murgia, M., Umiltà, C., \& Agostini, T. (2014). Automatic spatial association for luminance. Attention, Perception, \& Psychophysics, 76, 759-765. doi:10.3758/s13414013-0614-y

Gevers, W., Lammertyn, J., Notebaert, W., Verguts, T., \& Fias, W. (2006). Automatic response activation of implicit spatial information: Evidence from the SNARC effect. Acta Psychologica, 122, 221233. doi:10.1016/j.actpsy.2005.11.004

Hartmann, M., Gashaj, V., Stahnke, A., \& Mast, F. W. (2014). There is more than "more is up": Hand and foot responses reverse the vertical association of number magnitudes. Journal of Experimental Psychology: Human Perception and Performance, 40, 1401-1414.

Hartmann, M., Mast, F. W., \& Fischer, M. H. (2015). Spatial biases during mental arithmetic: Evidence from eye movements on a blank screen. Frontiers in Psychology, 6, 12. doi:10.3389/fpsyg.2015. 00012

Hayhoe, M., \& Ballard, D. (2005). Eye movements in natural behavior. Trends in Cognitive Sciences, 9, 188-194.

Holmes, K. J., \& Lourenco, S. F. (2011). Common spatial organization of number and emotional expression: A mental magnitude line. Brain and Cognition, 77, 315-323. 
Holmes, K. J., \& Lourenco, S. F. (2012). Orienting numbers in mental space: Horizontal organization trumps vertical. Quarterly Journal of Experimental Psychology, 65, 1044-1051.

Holmes, K. J., \& Lourenco, S. F. (2013). When numbers get heavy: Is the mental number line exclusively numerical? PLOS ONE, 8, e58381. doi:10.1371/journal.pone.0058381

Hung, Y., Hung, D. L., Tzeng, O. J.-L., \& Wu, D. H. (2008). Flexible spatial mapping of different notations of numbers in Chinese readers. Cognition, 106, 1441-1450.

Keren, G. B., \& Wagenaar, W. A. (1985). On the psychology of playing blackjack: Normative and descriptive considerations with implications for decision theory. Journal of Experimental Psychology: General, 114, 133-158. doi:10.1037/0096-3445.114.2.133

Klein, E., Huber, S., Nuerk, H.-C., \& Moeller, K. (2014). Operational momentum affects eye fixation behaviour. Quarterly Journal of Experimental Psychology, 67, 1614-1625.

Knops, A., Dehaene, S., Berteletti, I., \& Zorzi, M. (2014). Can approximate mental calculation account for operational momentum in addition and subtraction? Quarterly Journal of Experimental Psychology, 67, 1541-1556. doi:10.1080/17470218.2014.890234

Knops, A., Thirion, B., Hubbard, E. M., Michel, V., \& Dehaene, S. (2009). Recruitment of an area involved in eye movements during mental arithmetic. Science, 324, 1583-1585.

Knops, A., Viarouge, A., \& Dehaene, S. (2009). Dynamic representations underlying symbolic and nonsymbolic calculation: Evidence from the operational momentum effect. Attention, Perception, \& Psychophysics, 71, 803-821. doi:10.3758/APP.71.4.803

Loetscher, T., Bockisch, C. J., Nicholls, M. E., \& Brugger, P. (2010). Eye position predicts what number you have in mind. Current Biology, 20, R264-R265.

Loetscher, T., Bockisch, C. J., \& Brugger, P. (2008). Looking for the answer: The mind's eye in number space. Neuroscience, 151, 725729.

Loetscher, T., Schwarz, U., Schubiger, M., \& Brugger, P. (2008). Head turns bias the brain's internal random generator. Current Biology, 18, R60-R62.

Loftus, G. R., \& Mackworth, N. H. (1978). Cognitive determinants of fixation location during picture viewing. Journal of Experimental Psychology: Human Perception and Performance, 4, 565-572. doi: 10.1037/0096-1523.4.4.565

Marghetis, T., Nuñez, R., \& Bergen, B. K. (2014). Doing arithmetic by hand: Hand movements during exact arithmetic reveal systematic, dynamic spatial processing. Quarterly Journal of Experimental Psychology, 67, 1579-1596.
Masson, N., \& Pesenti, M. (2014). Attentional bias induced by solving simply and complex addition and subtraction problems. Quarterly Journal of Experimental Psychology, 67, 1514-1526. doi:10.1080/ 17470218.2014 .903985

McCarthy, A., Lee, K., Itakura, S., \& Muir, D. W. (2006). Cultural display rules drive eye gaze during thinking. Journal of Cross-Cultural Psychology, 37, 717-722.

McCrink, K., Dehaene, S., \& Dehaene-Lambertz, G. (2007). Moving along the number line: Operational momentum in nonsymbolic arithmetic. Perception \& Psychophysics, 69, 1324-1333.

Oldfield, R. C. (1971). The assessment and analysis of handedness: The Edinburgh inventory. Neuropsychologia, 9, 97-113. doi:10.1016/ 0028-3932(71)90067-4

Pinhas, M., \& Fischer, M. H. (2008). Mental movements without magnitude? A study of spatial biases in symbolic arithmetic. Cognition, 109, 408-415.

Proctor, R. W., \& Cho, Y. S. (2006). Polarity correspondence: A general principle for performance of speeded binary classification tasks. Psychological Bulletin, 132, 416-442. doi:10.1037/0033-2909. 132.3.416

Rinaldi, L., Brugger, P., Bockisch, C. J., Bertolini, G., \& Girelli, L. (2015). Keeping an eye on serial order: Ocular movements bind space and time. Cognition, 142, 291-298.

Santens, S., \& Gevers, W. (2008). The SNARC effect does not imply a mental number line. Cognition, 108, 263-270.

Santiago, J., \& Lakens, D. (2015). Can conceptual congruency effects between number, time, and space be accounted for by polarity correspondence? Acta Psychologica, 156, 179-191. doi:10.1016/j. actpsy.2014.09.016

Shaki, S., \& Fischer, M. H. (2008). Reading space into numbers: A crosslinguistic comparison of the SNARC effect. Cognition, 108, 590599. doi:10.1016/j.cognition.2008.04.001

van Dijck, J.-P., \& Fias, W. (2011). A working memory account for spatial-numerical associations. Cognition, 119, 114-119. doi:10. 1016/j.cognition.2010.12.013

Wiemers, M., Bekkering, H., \& Lindemann, O. (2014). Spatial interferences in mental arithmetic: Evidence from the motion-arithmetic compatibility effect. Quarterly Journal of Experimental Psychology, 67, 1557-1570.

Winter, B., \& Matlock, T. (2013). More is up ... right: Random number generation along two axes. In M. Knauff, N. Pauen, N. Sebanz, \& I. Wachsmuth (Eds.), Proceedings of the 35th Annual Conference of the Cognitive Science Society (pp. 3789-3794). Austin, TX: Cognitive Science Society. 